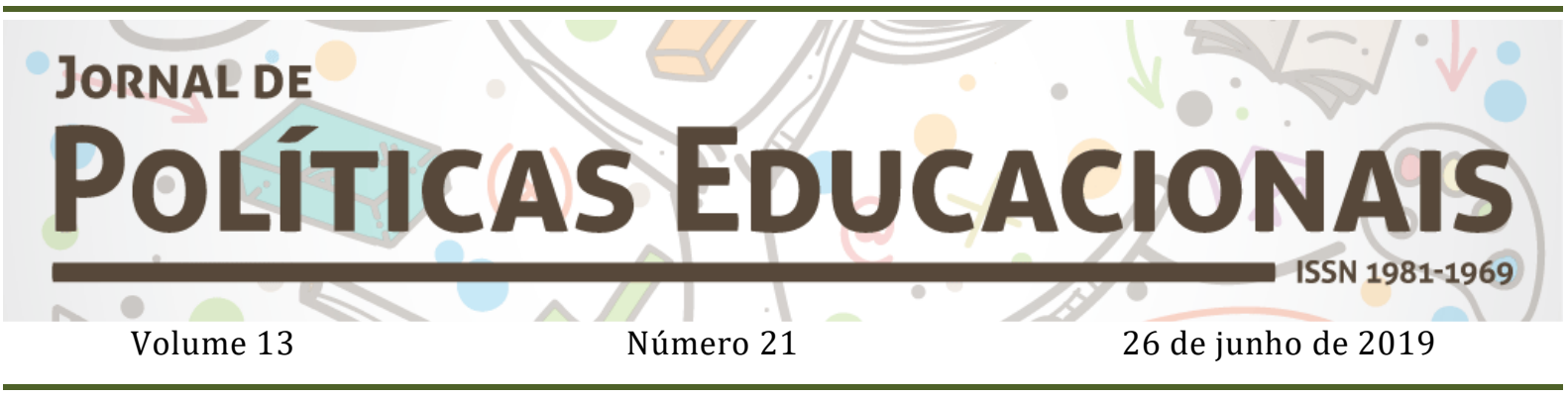

\title{
Estudos de revisão sobre produção acadêmica em políticas educacionais (2000-2010) ${ }^{1}$
}

\section{Review studies on academic production in educational policies (2000- 2010)}

\section{Estudios de revisión sobre producción académica en políticas educativas (2000-2010)}

\author{
Márcia Aparecida Jacomini ${ }^{2}$ \\ Marieta Gouvêa de Oliveira Penna ${ }^{3}$ \\ Isabel Melero Bello ${ }^{4}$
}

Citação: JACOMINI, M. A.; PENNA, M. G. de O.; BELLO, I. M. Estudos de revisão sobre produção acadêmica em políticas educacionais (2000-2010). Jornal de Políticas Educacionais. V. 13, n. 21. Junho de 2019.

http://10.5380/jpe.v12i0.64012

\begin{abstract}
Resumo:
Neste artigo são analisadas dez teses e dissertações que estudaram a produção acadêmica em políticas educacionais no Brasil no período de 2000-2010, selecionadas de um banco com 1.283 trabalhos coletados no Banco de Teses da Capes. A questão que norteia a pesquisa é saber quais tipos de revisão foram privilegiados pelos autores, com o objetivo de classificar as produções a partir da análise dos objetivos e
\end{abstract}

\footnotetext{
${ }^{1}$ Pesquisa financiada pelo CNPq, Edital Universal 14/2014.

${ }^{2}$ Doutora em Educação pela Faculdade de Educação da Universidade de São Paulo (2008). Professora Associada de graduação e pós-graduação do Departamento de Educação da Escola de Filosofia, Letras e Ciências Humanas da Universidade Federal de São Paulo (Unifesp) - Campus Guarulhos.

${ }^{3}$ Possui Bacharelado e Licenciatura em História pela Universidade de São Paulo (1985), Mestrado (2003), Doutorado (2007) Pós-Doutorado (2016) em Educação: História, Política, Sociedade: Educação e Ciências Sociais pela Pontifícia Universidade Católica de São Paulo.

${ }^{4}$ Possui graduação em Pedagogia pela Universidade de São Paulo (1994), mestrado em Educação: História, Política, Sociedade pela Pontifícia Universidade Católica de São Paulo (2003) e doutorado em Educação: Didática, Teorias de Ensino e Práticas Escolares pela Universidade de São Paulo (2008). Professora adjunta da Escola de Filosofia, Letras e Ciências Humanas da Universidade Federal de São Paulo do curso de Pedagogia e da pós-graduação em Educação (stricto sensu) na área de Teorias do Currículo e Formação de Professores.
} 
das metodologias adotadas. Tomou-se como aporte teórico autores que têm pesquisado/problematizado os estudos de revisão. Os textos foram classificados em estudos de revisão que mapeiam e estudos de revisão que avaliam e sintetizam. Conclui-se que há a necessidade de maior sistematização das características e denominações dos estudos de revisão, a fim de orientar as pesquisas desse tipo a delinearem a abordagem metodológica de maneira clara, na área da pesquisa educacional em sentido amplo e no domínio dos estudos que se voltam à compreensão e problematização das políticas públicas do setor da educação escolar, em particular.

Palavras-chaves: Estudos de Revisão; Políticas Educacionais; Produção Acadêmica.

\section{Abstract:}

In this article we analyze 10 theses and dissertations that studied the academic production in educational policies in Brazil, in the period 2000-2010, selected from a bank with 1.283 works collected in the Bank of Thesis of Capes. The question that guides the research is to know which types of revision were privileged by the authors in order to classify the researches based on the analysis of the objectives and the methodologies adopted. It was taken as theoretical referential authors who have researched/problematized the review studies. The texts were classified in reviewing studies that map and review studies that evaluate and synthesize. It is concluded that there is a need for a greater systematization of the characteristics and denominations of the review studies, in order to help the researches of this type to delineate the methodological approach in a clear way, in the area of educational research in a broad sense and in the field of studies that are focused on understanding and problematizing public policies in the school education sector, in particular.

Keywords: Educational Policies; Review Studies; Academic Production.

\section{Resumen:}

En este artículo se analizan 10 tesis y disertaciones que estudiaron la producción académica en políticas educativas en Brasil en el período 2000-2010, seleccionadas de un banco con 1.283 trabajos recogidos en el Banco de Tesis da Capes. La cuestión que orienta la investigación es saber qué tipos de revisión fueron privilegiados por los autores, con el objetivo de clasificar las producciones a partir del análisis de los objetivos y de las metodologías adoptadas. Se tomaron como aporte teórico autores que han investigado/problematizado los estudios de revisión. Los textos fueron clasificados en estudios de revisión que mapean y estudios de revisión que evalúan y sintetizan. Se concluye que hay la necesidad de mayor sistematización de las características y denominaciones de los estudios de revisión a fin de orientar las investigaciones de este tipo a delinear el enfoque metodológico de manera clara en el área de la investigación educativa en sentido amplio y en el ámbito de los estudios que se vuelven a la comprensión y problematización de las políticas públicas del sector de la educación escolar, en particular.

Palabras claves: Estudios de revisión; Políticas Educativas; Producción Académica.

\section{Introdução}

A criação do Sistema Nacional de Pós-Graduação em $1965^{5}$ foi um marco importante na institucionalização da pesquisa em educação. Nesses mais de 50 anos, o número de teses e dissertações na área vem crescendo, concomitantemente ao aumento dos programas de pós-graduação. Em 2016, eram 177 programas e 246 cursos de pósgraduação em educação, incluso o mestrado profissional (BRASIL, 2016).

Esse aumento na produção acadêmica tem sido acompanhado com atenção pelos pesquisadores, com vistas à consolidação da área. Como parte da reflexão sobre a produção acadêmica na área educacional, alguns trabalhos que têm indicado fragilidades

\footnotetext{
${ }^{5}$ Parecer no 977., de 3 de dezembro de 1965.
} 
acerca das questões teórico-metodológicas, ressaltando-as como aspectos fundamentais para fazer avançar o conhecimento em educação. Entre eles, destacam-se o estudo de Warde (1990) sobre o papel da pesquisa na pós-graduação em educação, alertando para certo sincretismo teórico e abandono do método nos trabalhos dos pós-graduandos, e o de Gatti (2012) sobre as dificuldades de caracterização do campo da educação, frente à sua polissemia, às ambiguidades e à complexidade típica de uma área com muitas subáreas, o que dificulta a construção metodológica das pesquisas.

Moraes (2001) e Borba e Valdemarin (2010), ao analisarem a produção científica no campo da educação, também destacam o que denominam de um recuo da teoria nas pesquisas, enfatizando a teorização como necessária à construção do objeto, de forma a possibilitar a análise e compreensão do fenômeno estudado. Alves-Mazzotti (2001, p. 40), a fim de reafirmar a importância da teorização na pesquisa educacional, recorre a resultados de estudos sobre a qualidade de tal produção que, segundo a autora, indicam as seguintes deficiências:

(a) pobreza teórico-metodológica na abordagem dos temas, com grande número de estudos puramente descritivos e/ou "exploratórios"; (b) pulverização e irrelevância dos temas escolhidos; (c) adoção acrítica de modismos na seleção de quadros teórico-metodológicos; (d) preocupação com a aplicabilidade imediata dos resultados; e (e) divulgação restrita dos resultados e pouco impacto sobre as práticas.

Como afirma Brandão (2002), trata-se de campo em construção que se consolida, entre outros aspectos, a partir da produção explicitada em teses e dissertações, periódicos especializados, eventos, o que evidencia relevância de estudos como o que aqui se propõe. De todo modo, concorda-se com Charlot (2006, p. 9), ao enfatizar que "0 que é específico da educação como área de saber é o fato de ela ser uma área na qual circulam, ao mesmo tempo, conhecimentos (por vezes de origens diversas), práticas e políticas". A nosso ver, tal especificidade requer permanente vigilância epistemológica, tal como formulado por Bourdieu, Passeron, Chamboredon (2004), por se tratar de área de conhecimento na qual circulam conceitos e métodos provenientes de outros campos disciplinares, e que tem por objeto a educação, aqui entendida como prática social, portanto, entrecortada por saberes, práticas, finalidades educativas.

Ainda como parte desse movimento, encontram-se os trabalhos que analisam teses, dissertações, artigos e livros sobre temáticas específicas, produzidos num 
determinado período, e que lançam luz acerca das características e das contribuições da produção acadêmica (WITTMANN; GRACINDO, 2001; MARTINS, 2011; AUTOR, 2014).

De acordo com Stremel (2016), a política educacional emerge como campo específico a partir da década de 1960 e encontra-se, no momento, em fase de expansão e estabilização. Considera, contudo, que a consolidação desse campo acadêmico demanda melhor definição dos objetos de estudo, ampliação do referencial teórico para análise das políticas, da conceituação de política educacional, da internacionalização dos estudos e da ampliação da interlocução com outras áreas, tais como as ciências políticas, sociais, econômicas e com a teoria social, em sentido amplo.

Em análise sobre o estado do campo da pesquisa em políticas públicas, Souza (2003) indica que a escassa acumulação de conhecimento, a predominância de estudos setoriais e a proximidade com os órgãos de governo são alguns problemas que precisam ser superados para sua consolidação enquanto campo de conhecimento científico.

O aumento da produção acadêmica no campo da educação e os desafios apresentados indicam relevância de estudos como o que aqui se apresenta, a fim de contribuir para o autoconhecimento da área e sua consolidação.

Nesse contexto, duas pesquisas ${ }^{6}$ recentes estudaram a produção acadêmica em políticas educacionais, no período de 2000 a 2010. A primeira trabalhou com os dados disponíveis no Banco de Teses da Coordenação de Aperfeiçoamento de Pessoal de Nível Superior (Capes) mediante a leitura dos resumos de 1.283 teses e dissertações coletadas no ano de 2012, em programas de pós-graduação com nota igual ou superior a cinco, na avaliação da Capes, no triênio que se encerrou em 2010. Corroborou para a definição do critério de seleção a consideração de que nesses programas está parte expressiva do que tem sido produzido sobre e em políticas educacionais no país. Na segunda pesquisa, foram estudados 22 trabalhos, dessa mesma base, classificados no eixo Abordagens teóricometodológicas. Desses 22 trabalhos, 10 deles se caracterizam como estudos de revisão, os quais serão analisados neste artigo. A questão que norteia a pesquisa é saber quais tipos de estudo de revisão foram privilegiados pelos autores dos dez trabalhos, com o objetivo de classificar as teses e dissertações a partir da análise dos objetivos e das metodologias adotadas pelos pós-graduandos.

\footnotetext{
${ }^{6}$ A primeira foi financiada pelo CNPq/Capes, Edital 07/2011, e a segunda pelo CNPq, Edital Universal 14/2014.
} 
O artigo está dividido em três tópicos além desta introdução. No primeiro, discutem-se os diferentes tipos de pesquisas que têm como objeto de estudo a produção acadêmica, busca-se, com base na literatura, conhecer as características desses trabalhos. Seguida a esse marco teórico, serão analisadas dez teses e dissertações com base na abordagem metodológica e nos objetivos em diálogo com o referencial do primeiro tópico. Nas considerações finais, debate-se o papel desse tipo de pesquisa na constituição do campo acadêmico da política educacional.

\section{Estudos de revisão da produção acadêmica: características e delimitações}

Os estudos de revisão da produção acadêmica/científica têm se tornado cada vez mais necessários na medida em que indicam como um campo de saber vem se desenvolvendo ao sistematizarem o conhecimento produzido. Entre os estudos que investigam a produção acadêmica, há diferentes denominações para se referir a pesquisas semelhantes, mas há também diferentes abordagens de investigação (VOSGERAU; ROMANOWSKI, 2014; ANDRÉ et al., 1999; GATTI, 2012; THOMAS, 2007).

A despeito dos distintos posicionamentos sobre a prática baseada em evidência na educação (THOMAS, 2007), considera-se que, em meio à expansão das pesquisas acadêmicas, a realização de estudos de mapeamento, avaliações e análises críticas que indicam o estado do conhecimento da produção é fundamental ao direcionamento das novas pesquisas. Trata-se de conhecer a própria produção e enfrentar os desafios que vêm sendo sinalizados. De acordo com Vosgerau e Romanowski (2014, p. 167),

Os estudos de revisão consistem em organizar, esclarecer e resumir as principais obras existentes, bem como fornecer citações completas abrangendo o espectro de literatura relevante em uma área. As revisões de literatura podem apresentar uma revisão para fornecer um panorama histórico sobre um tema ou assunto considerando as publicações em um campo. Muitas vezes uma análise das publicações pode contribuir na reformulação histórica do diálogo acadêmico por apresentar uma nova direção, configuração e encaminhamentos.

Assim, consideram-se como estudos de revisão, de forma ampla, todos aqueles que tomam como objeto de pesquisa a produção científica/acadêmica de determinada área, subárea ou campo de conhecimento, tendo em conta a especificidade do recorte temático e temporal. Dentro desse espectro amplo de estudos de revisão, são apresentadas diferentes denominações e formas de realizar os estudos. 
Ao analisarem trabalhos que tratam de estudos de revisão da produção acadêmica publicados em periódicos nacionais e internacionais, Vosgerau e Romanowski (2014) encontraram várias formas de realização das pesquisas. As autoras classificaram os estudos por elas localizados em dois tipos: revisões que mapeiam e revisões que avaliam e sintetizam.

Entre as revisões que mapeiam, cujas características centrais são a realização da sistematização e da indicação do que caracteriza determinado conjunto de produções acadêmicas, encontram-se os estudos denominados levantamento bibliográfico, revisão de literatura, revisão bibliográfica, estudo bibliométrico e estado da arte ou estado do conhecimento (VOSGERAU; ROMANOWSKI, 2014).

Os estudos de revisão que mapeiam podem ser divididos em três tipos, ressalvando que as fronteiras entre eles não são totalmente claras. No primeiro tipo, encontram-se os trabalhos que se caracterizam pelo mapeamento e apresentação da produção de forma inventariante e descritiva, a exemplo de levantamento bibliográfico (MORAIS; ASSUMPÇÃO, 2012, e SANTOS, 2013) e de estudo bibliométrico (MARCELO; HAYASHI, 2013). Esse último tem como objetivo elaborar índices de produção do conhecimento científico, verificar indicadores de produção científica, indicadores de citações, indicadores de ligação (coautoria).

O segundo tipo congrega as revisões que, além do levantamento das características gerais dos trabalhos estudados, analisam a produção, classificam-nas e apresentam as principais conclusões, tendo em vista a sistematização dos achados das pesquisas que compõem o escopo do estudo. Entre eles, destacam-se a revisão de literatura (MIRANDA; FARIAS, 2009), a revisão bibliográfica (FRANÇA; MALTA; ALVES, 2012) e a revisão de produção acadêmica (JACOMINI, 2014). Embora com denominações diferentes, esses estudos apresentam características semelhantes na forma de conduzir a pesquisa. São, geralmente, estudos que analisam a produção em suas características gerais e, principalmente, em relação às conclusões, com o objetivo de informar o que se sabe acerca de uma determinada temática, campo ou área de conhecimento.

No terceiro tipo, estão as pesquisas estado da arte ou estado do conhecimento, mais correntes na área de educação. Entre os estudos que se classificam como estado da arte, há diferenças no recorte e na maneira como são realizadas as análises, contudo, constitui objetivo comum dessas pesquisas apresentar informações sobre as 
características e contribuições da produção que compõem o escopo do estudo, de maneira a informar o estado do conhecimento numa área ou campo de saber.

De acordo com Nóbrega-Therrien e Therrien (2004), as pesquisas do tipo estado da arte têm como objetivo mapear e discutir a produção acadêmica de determinada área ou campo de conhecimento.

Para Ferreira (2002, p. 258), as pesquisas denominadas estado da arte ou estado do conhecimento

[...] parecem trazer em comum o desafio de mapear e discutir uma certa produção acadêmica em diferentes campos do conhecimento, tentando responder que aspectos e dimensões vêm sendo destacados e privilegiados em diferentes épocas e lugares, de que formas e em que condições têm sido produzidas certas dissertações de mestrado, teses de doutorado, publicações em periódicos e comunicações em anais de congressos e de seminários. Também são reconhecidas por realizarem uma metodologia de caráter inventariante e descritivo da produção acadêmica e científica sobre o tema que busca investigar, à luz de categorias e facetas que se caracterizam enquanto tais em cada trabalho e no conjunto deles, sob os quais o fenômeno passa a ser analisado.

Embora as pesquisas do tipo estado da arte tenham características parecidas às pesquisas de revisões de literatura e bibliográfica, aquelas se diferenciam destas pela abrangência do escopo da pesquisa, pelo tipo de análise que realizam e pela finalidade de indicar as características e contribuições de determinada produção acadêmica.

Uma questão debatida quando se trata desse tipo de pesquisa é a utilização apenas dos resumos nas análises e caracterização das pesquisas. Ferreira (2002) advoga que há limitações para o uso apenas dos resumos nas pesquisas do tipo estado da arte, principalmente quando o pesquisador, entre outros aspectos analisados, propõe-se a classificar os trabalhos em determinado agrupamento no que se refere à metodologia, à teoria e até mesmo ao tema.

Os estudos de revisão que avaliam e sintetizam caracterizam-se pela adoção de critérios de inclusão e exclusão com base na temática, mas também na qualidade metodológica das pesquisas inventariadas (VOSGERAU; ROMANOWSKI, 2014). Nesse tipo de revisão, a análise central recai sobre a sistematização dos resultados, por isso é fundamental garantir a qualidade metodológica, de forma que se possa comparar os resultados dos estudos. Entre eles, destacam-se a revisão integrativa de literatura (SOBRAL; CAMPOS, 2012), as revisões sistemáticas (DAVIES, 2007) e a síntese sistemática de pesquisa (GOUGH, 2007), que têm como objetivo contribuir com as políticas e práticas 
à medida que identificam e analisam as evidências de pesquisas acumuladas. Também são classificadas como revisão que avaliam e sintetizam, de acordo com Vosgerau e Romanowski (2014), a síntese de evidências qualitativas, a metassíntese qualitativa, a meta-análise e a metassumarização, não analisadas neste artigo.

No campo acadêmico das políticas educacionais $s_{2}$ há autores que têm destacado a importância de pesquisas de revisão que tenham como propósito compreender o desenho epistemológico, teórico e metodológico da produção acadêmica. Com a denominação de metapesquisa, Mainardes e Tello (2016) classificam as revisões que buscam identificar como os pesquisadores articulam as questões epistemológicas, as teorias e os conceitos que fundamentam a pesquisa. A metapesquisa visa identificar a perspectiva e o posicionamento epistemológico, o enfoque epistemetodológico, o tipo de pesquisa (natureza teórica, pesquisa empírica, comentários ou críticas) e os marcos teóricos (conceitos, os níveis de abordagem e abstração) (MAINARDES; TELLO, 2016).

Os autores destacam três níveis de abordagem e abstração que podem ser considerados na análise das pesquisas acadêmicas. No nível da descrição, encontram-se as pesquisas de caráter predominantemente descritivo, com poucas análises e discussão dos dados apresentados. No nível da análise, estão os estudos que garantem integração entre teoria e dados, o que permite maior nível de generalidade. E como nível mais elevado de abstração, encontra-se a compreensão, que engloba estudos que, além da descrição e das análises, apresentam explicações com base em interpretações. Nesses estudos, percebe-se articulação e coerência entre perspectiva epistemológica, posicionamento epistemológico e enfoque epistemetodológico (MAINARDES; TELLO, 2016).

A metapesquisa se distingue das pesquisas de revisão apresentadas anteriormente na medida em que, enquanto aquelas têm como objetivo sistematizar e sintetizar os resultados e as características das pesquisas sobre uma área, campo de conhecimento ou determinado tema, a metapesquisa visa explorar os fundamentos epistemológicos, teóricos, metodológicos, os objetivos, as questões de pesquisa, entre outros.

A partir desse referencial sobre pesquisas de revisão, na próxima seção são analisadas as dez teses e dissertações, de modo a identificar o tipo de estudo de revisão realizado, com base na abordagem metodológica e nos objetivos, especialmente, em relação à abrangência do estudo e o tipo de análise realizada. Mas é preciso destacar que 
se trata de um exercício didático, posto que há muitas semelhanças entre estudos com denominações diferentes.

\section{Tipos de estudos de revisão}

As teses e dissertações que compõem o escopo deste artigo foram produzidas em cinco instituições de ensino superior, a saber: Universidade Federal de São Carlos (2), Universidade Estadual Paulista - Marília (4), Universidade Federal do Rio de Janeiro (1), Universidade Metodista de Piracicaba (1), Pontifícia Universidade Católica do Rio de Janeiro (1) e Universidade de São Paulo (1). As defesas ocorreram nos seguintes anos: 2009, 2004 e 2008, dois trabalhos em cada ano; 2010, 2003, 2009 e 2007, um trabalho em cada ano. As cinco teses e cinco dissertações analisadas estão vinculados a sete diferentes linhas de pesquisa, sendo três trabalhos pertencentes à mesma linha, e foram orientadas por dez diferentes professores.

A análise dos trabalhos foi precedida da leitura integral dos textos e de preenchimento de ficha analítica que compreende informações sobre o trabalho, tais como: objetivos, objeto de estudo, problema de pesquisa, organização acerca da perspectiva epistemológica, do referencial teórico, do método, dos procedimentos metodológicos, conclusões e contribuição ao debate. Para a escrita deste artigo, o foco das análises recaiu sobre os elementos constituintes dos trabalhos que os caracterizam como estudos de revisão, ou seja, aqueles que tiveram como objeto de estudo uma determinada produção acadêmica. Buscou-se, a partir do objeto de estudo, dos objetivos e dos procedimentos metodológicos da pesquisa, indicar o tipo de revisão empreendida, quando não nomeada pelo pesquisador. Os dez trabalhos estudaram temas específicos, vinculados à área da educação e ao campo das políticas educacionais. Uma característica comum a todos eles é que tiveram como objeto de estudo algum tipo de produção acadêmica: teses, dissertações, artigos, livros, anais de eventos, relatórios de pesquisa, produção de entidades etc. Entre os objetivos, constam os propósitos de mapear, conhecer, classificar, sistematizar e analisar a produção que compõe o escopo de cada pesquisa. Por isso, foram considerados estudos de revisão.

Como aspecto que perpassa todas as pesquisas, encontra-se a apresentação das características gerais e das contribuições dos trabalhos analisados, em forma de sistematização da produção sobre determinada temática, produzida em período e local 
delimitados, com indicação de aspectos a serem investigados em pesquisas futuras. Essa contribuição geral das pesquisas foi realizada de formas diversas e com níveis diferentes de aprofundamento e de especificação da produção estudada.

Com base na classificação de Vosgerau e Romanowski (2014), nove trabalhos são do tipo de revisão que mapeia e um do tipo de revisão que avalia e sintetiza. Entre as nove revisões do tipo que mapeia, três autores autodenominaram os respectivos trabalhos como: estado da arte (1), pesquisa tipo estado da arte (1) e pesquisa sobre pesquisa (1). Dos seis não autodenominados, consideraram-se, pelas características apresentadas, que dois são do tipo estado da arte, três revisões de literatura ou bibliográfica e um levantamento bibliográfico. Um único trabalho de revisão do tipo que avalia e sintetiza se autodenominou como revisão sistemática, usando, como referencial, Davies (2007).

Segue-se apresentação de características das teses e dissertações conforme o tipo de estudo de revisão.

\section{Estudos de revisão que mapeiam - estado da arte e pesquisa sobre pesquisa}

Os trabalhos de Gregório (2009), Mauá Júnior (2007), Gonçalves (2003), Maia (2004) e Carvalho (2004), classificados como estudos de revisão que mapeiam, tipo estado da arte ou pesquisa sobre pesquisa, trazem em comum análises com foco nas contribuições de produções acadêmicas que pesquisaram as seguintes temáticas: educação comparada, planejamento escolar, relação entre Estado e Educação, administração educacional e caracterização de quem pesquisa e como é pesquisada a escola de educação básica no Brasil, com o objetivo de indicar os aspectos e as dimensões que são destacados e privilegiados pelos pesquisadores.

Os trabalhos que compuseram o corpus das pesquisas desses autores foram produzidos entre 1961 e 2006, nos seguintes períodos: 1987-2006, 1961-2005, 19712000, 1983-2000, 1996-2000. Com exceção de Carvalho (2004), cuja pesquisa abrangeu um curto período de quatro anos, os demais estabeleceram como recorte temporal mais de quinze anos. Nesse sentido, pode-se dizer que, em termos de época, eles retratam características de pesquisas produzidas, principalmente, da segunda metade do século XX e início dos anos 2000. Os trabalhos analisados pelos cinco autores foram coletados nos seguintes repositórios: Banco de teses da Capes, bibliotecas de universidades do estado de São Paulo, periódicos e anais de eventos, arquivo da Associação Brasileira de Política e 
Administração da Educação, site da Capes e CNPq (relatórios de pesquisas financiadas por estas agências de fomento).

Em relação à quantidade de trabalhos que cada pesquisador analisou, encontramos bastante diversidade: Gregório (2009) analisou 11 teses e dissertações, e Mauá Júnior (2007), seis; Gonçalves (2003) analisou 202 artigos, e Maia (2004), quatro números da revista Cadernos de Administração (1961-1968) e três da Revista Brasileira de Política e Administração da Educação (1983-2000). Não há informação sobre quantos relatórios de pesquisas financiadas pela Capes e CNPq foram analisados por Carvalho (2004). Todos os autores leram os referidos trabalhos na íntegra.

Em relação às contribuições desses estudos de revisão, destacamos a sistematização dos dilemas e reflexões históricas sobre educação comparada (GREGÓRIO, 2009); a discussão sobre a importância de as pesquisas de revisão se debruçarem sobre a análise das metodologias, das bases teóricas e das referências bibliográficas utilizadas pelos autores, e o mapeamento da produção sobre o tema da relação Estado e Educação (GONÇALVES, 2003); a indicação das principais características da produção sobre administração escolar (MAIA, 2004); a indicação das tendências das pesquisas, realizadas por professores universitários e financiadas pela Capes e pelo CNPq (CARVALHO, 2004).

Esses cinco trabalhos, mesmo que de forma distinta, com maior ou menor extensão e profundidade, tiveram um caráter inventariante e descritivo da produção acadêmica analisada, sendo que Gonçalves (2003) adotou os procedimentos de análise de conteúdo de Bardin (2004) (pré-análise, exploração do material e tratamento dos resultados). Destaca-se, ainda, o fato de os cinco autores terem apresentado e analisado as conclusões dos trabalhos e indicado as principais contribuições, característica importante de estudos de revisão do tipo estado da arte.

Dos autores, a que mais detalhou os procedimentos metodológicos foi Gonçalves (2003). Com o objetivo de mapear, caracterizar e analisar a produção acadêmica que trata da relação entre Educação e Estado, a autora buscou identificar tendências, divergências, consensos e movimentos que permitissem uma compreensão mais clara das perspectivas, lacunas e limitações dos artigos analisados. Tomou como referência Prost (1996), para quem o corpus analisado deve ter três características básicas: ser contrastivo de forma a permitir comparações; ser diacrônico para recuperar continuidades e viradas; ser significativo de situações de comunicação determinadas. 
Por outro lado, ao trabalho de Mauá Junior (2007), apesar da autodenominação de pesquisa tipo estado da arte, parece faltar à investigação alguns aspectos importantes a esse tipo de estudo. Não houve aprofundamento em analisar, categorizar e revelar os múltiplos enfoques da produção estudada, aspectos essenciais num estudo do tipo estado da arte (VOSGERAU; ROMANOWSKI, 2014). A pesquisa parece estar mais próxima de uma revisão da literatura ou bibliográfica.

Assim, nessas teses e dissertações, além da análise das características gerais dos trabalhos que compuseram o escopo das pesquisas, também houve análise dos objetivos, dos resultados, do tipo de pesquisa, dos temas, do referencial teórico e de tendências metodológicas, o que permitiu a indicação de características e tendências das produções estudadas, correspondendo, portanto, a estudos de revisão tipo estado da arte ou do conhecimento.

\section{Estudos de revisão que mapeiam - revisões de literatura e bibliográfica e levantamento bibliográfico}

Os trabalhos de Oliveira (2006), Grello (2008), Calixto (2010), Yanaguita (2008) pesquisaram as seguintes temáticas: estratégicas político-educativas concebidas e implementadas pelo Instituto Superior de Estudos Brasileiros (ISEB), a produção do Núcleo de Pesquisa sobre Ensino Superior (NUPES/USP), a formação do administrador escolar e a produção e circulação de saberes sobre financiamento da educação.

Em relação ao desenho metodológico, eles se aproximam dos cinco trabalhos anteriores no que se refere ao mapeamento que realizaram das produções sobre as temáticas pesquisadas, mas diferem, principalmente, pela ausência de uma análise abrangente do corpus das pesquisas envolvendo objetivos, resultados, tipo de pesquisa, referencial teórico e procedimentos metodológicos. 0 foco principal desses trabalhos foi a sistematização da produção estudada de forma inventariante.

Os trabalhos analisados foram produzidos em períodos mais curtos que aqueles da maioria das pesquisas classificadas como estado da arte. Oliveira (2006) pesquisou a produção do Instituto Superior de Estudos Brasileiros (ISEB), no período de 1955 a 1964, analisando as obras escritas entre 1958-1960, mas não informou o número de textos estudados. 0 objetivo da pesquisa foi compreender as estratégicas político-educativas concebidas e implementadas pelo Instituto. 0 período mais extenso de produção de 
trabalhos estudados foi de 17 anos, de 1988-2005, no qual Grello (2008) analisou 23 Cadernos de Pesquisa da Série Documentos de Trabalhos produzidos pelo Núcleo de Pesquisa sobre Ensino Superior (NUPES/USP). Além dos Cadernos, a autora também analisou artigos de membros do NUPES e editais do CNPq, com o intuito de verificar se a hipótese de que o Núcleo se antecipou às políticas educacionais para o ensino superior se confirmava. O levantamento bibliográfico realizado por Calixto (2010), sem indicação de período, consistiu na seleção de 16 livros, 31 artigos, 38 textos de legislação e 14 documentos de entidades relacionados à formação do administrador escolar, com a finalidade de identificar, sistematizar e analisar publicações que estudaram a formação desse profissional.

Esses três trabalhos, pelas características de identificar, sistematizar e analisar determinada produção acadêmica, foram considerados revisão de literatura ou revisão bibliográfica. As principais contribuições dessas pesquisas recaem sobre a sistematização da produção sobre o ideário educativo do ISEB, da produção do NUPES e sobre o conhecimento acerca da formação do administrador, do ponto de vista da legislação, das entidades nacionais e de textos acadêmicos.

Com a finalidade de compreender e analisar a produção e circulação de saberes sobre financiamento da educação, Yanaguita (2008) realizou pesquisa, por ela denominada histórica, como base no referencial da História Cultural. A autora analisou 41 livros publicados no período de 1991 a 2005, nos aspectos referentes à produção e circulação do conhecimento sobre financiamento da educação, considerando o mercado editorial e o campo educacional no qual estavam inseridos os autores. A pesquisa traz importantes contribuições sobre o processo de produção e circulação de conhecimento na área de educação, notadamente sobre financiamento da educação. Essa pesquisa não apresenta uma análise da produção sobre determinado tema ou campo de conhecimento. Contudo, pode-se dizer que a autora fez um levantamento bibliográfico, com indicação das características gerais dos livros analisados, mas cujo foco de análise recai sobre a compreensão do processo de produção e circulação do conhecimento sobre financiamento da educação.

\section{Estudos de revisão que avaliam e sintetizam - revisão sistemática}


O trabalho de Contiero (2009) teve como objetivo verificar as contribuições e os resultados dos estudos sobre a progressão continuada no estado de São Paulo. A pesquisa buscou identificar a origem dos trabalhos, o tipo de pesquisa, os temas investigados, os resultados e as tendências. Pretendia, também, analisar os aspectos teóricometodológicos, o que não foi possível devido à leitura apenas do resumo das teses e dissertações, conforme informou o autor. Os trabalhos foram coletados no banco de teses da Capes, com o uso do descritor "progressão continuada". Dos 113 trabalhos que emergiram na pesquisa inicial, após leitura do resumo, foram selecionados 54 que estudaram a temática da progressão continuada no estado de São Paulo. Para análise, as teses e dissertações foram classificadas em quatro categorias de acordo com os aspectos abordados: implantação do regime de progressão continuada, avaliação da aprendizagem no contexto da progressão continuada, o impacto do regime de progressão continuada na docência, a progressão continuada no marco da inclusão/exclusão/democratização do ensino. 0 autor utilizou a análise de conteúdo proposta por Bardin (1977) e dialogou com autores da área da educação e, mais especificamente, com autores que estudaram a organização do ensino em ciclos e a progressão continuada. Os achados e as conclusões da pesquisa contribuíram para elucidar que os enfoques principais dos estudos sobre progressão continuada são a avaliação da aprendizagem e a influência do regime de progressão continuada na docência. Quase 60\% dos trabalhos analisados tiveram esse enfoque. Ainda em relação às tendências, o autor informou não ter encontrado nenhum trabalho cujo foco tenha sido investigar se os alunos aprendem mais ou menos no regime de progressão continuada. Destacou que na avaliação dos trabalhos analisados, a progressão continuada assumiu feições de promoção automática. Por último, concluiu que há demanda em torno de uma formação dos professores que os qualifique melhor para trabalhar com as questões relacionadas ao processo ensino/aprendizagem/avaliação.

Observa-se pelas características do trabalho que o critério de inclusão/exclusão que caracteriza as revisões sistemáticas foi temático, o autor não usou o tipo de abordagem metodológica para selecionar os trabalhos ${ }^{7}$, posto que analisou todos aqueles que trataram do regime de progressão continuada no estado de São Paulo, mas o foco da pesquisa foi analisar e sistematizar os resultados de forma a apresentar a contribuição

\footnotetext{
${ }^{7}$ É importante lembrar, conforme visto na seção anterior, que a abordagem metodológica do trabalho é critério de seleção fundamental nos estudos de revisão sistemática.
} 
desses trabalhos para se pensar tanto as políticas educacionais quanto a formação de professores, além de evidenciar as tendências e lacunas dos estudos sobre a temática. De acordo com o autor,

[...] presente trabalho pode ser enquadrado na categoria de revisão sistemática (review systematic) cujo objetivo é elaborar a síntese de pesquisas realizadas por diferentes grupos de estudiosos para que se alcance uma análise mais aprofundada do conhecimento produzido sobre um determinado tópico (evidence based) (DAVIES, 2007). Mas trata-se de uma revisão exploratória e preliminar, pois tem o objetivo de mapear as pesquisas, conhecer as abordagens teórico-metodológicas mais utilizadas, as contribuições dos estudos, suas conclusões, demandas de pesquisa, entre outros. (CONTIERO, 2009, p. 21).

Com base nessa exposição do autor sobre o tipo de revisão empreendida e o que foi efetivamente realizado no trabalho, considerando que ele informou não ter realizado a leitura completa e a análise teórico-metodológica das teses e dissertações, e as análises apresentadas neste artigo sobre os diferentes tipos de estudos de revisão, poderíamos classificar a dissertação de Contiero como uma pesquisa tipo estado da arte.

\section{Algumas considerações para finalizar}

A classificação das dez teses e dissertações não foi tarefa fácil e pode apresentar imprecisões, posto que a maioria das pesquisas foi realizada sem a preocupação de seguir procedimentos específicos de determinado tipo de revisão. Não se trata, portanto, de uma classificação que encerra certezas, mas de um exercício de compreender como estão sendo produzidos os trabalhos de revisão, como parte de uma pesquisa mais ampla sobre produção acadêmica em políticas educacionais, em diálogo com a literatura que vem nomeando e indicando características desses estudos.

A análise permitiu verificar que a maior parte dos trabalhos é do tipo estudo de revisão que realiza mapeamento, conforme classificação de Vosgerau e Roamnowski (2014).

Entre os estudos de revisão que mapeiam, quatro são do tipo estado da arte, um autodenominado pesquisa sobre pesquisa, com características de estado da arte, três do tipo revisão bibliográfica ou de literatura e apenas um considerado do tipo levantamento bibliográfico. Entre os autodenominados estado da arte, consideramos que um se aproxima mais da revisão bibliográfica ou de literatura. 
Em relação às revisões que avaliam e sintetizam, observa-se carência de pesquisas. No universo deste estudo, somente um trabalho se autodenominou como revisão sistemática. Entrementes, observou-se entre os estudos de estado da arte proximidade à revisão sistemática e nesta proximidade com aqueles. Nesse sentido, observa-se também uma linha tênue que separa pesquisas que mapeiam e pesquisas que avaliam e sintetizam, especialmente no que se refere aos estudos de estado da arte.

Constatou-se ausência, no recorte desta pesquisa, de estudos do tipo metapesquisa (MAINARDES; TELLO, 2016). Considerando as características da metapesquisa de identificar a perspectiva e o posicionamento epistemológico, o enfoque epistemetodológico, o tipo de pesquisa e os marcos teóricos, seria interessante investir em estudo de revisão desse tipo. É possível que tal ausência se deva ao fato de que as discussões sobre metapequisa serem ainda recentes (TELLO; MAINARDES, 2015).

Um aspecto importante observado nesta pesquisa é o fato de os trabalhos analisados não serem apresentados como sendo estudos de revisão, embora no estabelecimento do objeto, do objetivo e na definição dos procedimentos metodológicos se caracterizarem como tal. Considera-se que, em certa medida, isso pode ser compreendido pelo fato de, na área da educação, o estabelecimento de características e delimitações de cada tipo de revisão ser processo ainda em construção. Exceção pode ser atribuída ao estado da arte ou estado do conhecimento, tipo de investigação mais conhecido entre os pesquisadores dessa área, mas que, ainda assim, apresenta limites tênues, no que se refere ao que vem sendo apresentado como revisões bibliográficas e de literatura.

Neste estudo, observou-se certa ausência de delimitações mais claras, com indicações de procedimentos de coleta e análises dos dados que justifiquem o uso de terminologias diferentes. Essas delimitações poderão orientar novas pesquisas e dar maior segurança aos pesquisadores na escolha do tipo de revisão a realizar, tendo em vistas os objetivos.

Em consideração ao crescimento da produção acadêmica em educação e, como parte desta, em políticas educacionais, faz-se cada vez mais necessário a realização de estudos de revisão para que possam mapear, sintetizar, analisar, avaliar e indicar características, tendências e lacunas da produção acadêmica, no sentido de informar novas pesquisas e contribuir para a análise crítica sobre o acúmulo de conhecimento da área. 
Entende-se que pesquisas de revisão que envolvam longos períodos, recortes amplos e que, além de mapear, avaliem e sintetizem resultados, com o objetivo de orientar novos estudos e contribuir para a formulação de políticas educacionais, demandam trabalho em rede, com o envolvimento de vários pesquisadores, pois, como sugere André (2001), o conhecimento sistemático e sistematizado da produção acadêmica na área da educação é tarefa coletiva e de longo prazo.

\section{Referências}

ANDRÉ, Marli. Pesquisa em educação: Buscando rigor e qualidade. Cadernos de Pesquisa, São Paulo, n. 113, p. 51-64, jul. 2001.

ANDRÉ, Marli et al. Estado da arte da formação de professores no Brasil. Educação \& Sociedade, Campinas, ano XX, n. 68, p. 301-309, dez. 1999.

BARDIN, Laurence. Análise de conteúdo. Lisboa: Edições 70, 2004.

BORBA, S.; VALDEMARIN, V. T. A construção teórica do real: uma questão para a produção do conhecimento em educação. Currículo sem Fronteiras, v.10, n.2, pp. 2337, jul./dez 2010.

BOURDIEU, P., CHAMBOREDON, J.-C., PASSERON, J.-C. Ofício de Sociólogo: metodologia da pesquisa na sociologia. Trad. Guilherme João de Freitas Teixeira. 4. ed., Petrópolis, RJ: Vozes, 2004.

BRANDÃO, Z. Pesquisa em educação: conversas com pós-graduandos. Rio de janeiro: Ed. PUC-Rio; São Paulo: Loyola, 2002.

BRASIL. Conselho Federal de Educação. Parecer CFE no 977, Brasília, DF, 1965.

BRASIL. Coordenação de Aperfeiçoamento de Pessoal de Nível de Ensino Superior. Dados Quantitativos de Programas Recomendados e reconhecidos. Brasília, DF, 2016. Disponível em:

<https://sucupira.capes.gov.br/sucupira/public/consultas/coleta/programa/quantitati vos/quantitativoAreaAvaliacao.jsf>. Aceso em: 22/02/2017.

CALIXTO, Eulália Araújo. A construção do conhecimento sobre formação do administrador escolar: uma análise a partir das publicações nacionais. Dissertação (Mestrado em Educação). Faculdade de Filosofia e Ciências, Universidade Estadual Paulista. Marília, 2010.

CARVALHO, Merise Santos de. A pesquisa educacional sobre a escola pública de ensino fundamental, nos projetos e relatórios de pesquisa elaborados por docentes das universidades brasileiras. Tese (Doutorado em Educação). Universidade federal do Rio de Janeiro, 2004. 
CHARLOT, Bernard. A pesquisa educacional entre conhecimentos, políticas e práticas: especificidades e desafios de uma área de saber. Revista Brasileira de Educação, v. 11 n. 31, p. 7-18, jan./abr. 2006.

CONTIERO, Alex Sander. 0 regime de progressão continuada no estado de São Paulo: indicadores e perspectivas para novas pesquisas. Dissertação (Mestrado em Educação). Universidade Metodista de Piracicaba, Piracicaba, 2009.

DAVIES, Philip. Revisões sistemáticas e a Campbell Collaboration. In: THOMAS, Gary;

PRING, Richard (Orgs). Educação baseada em evidências: a atualização dos achados científicos para a qualificação da prática pedagógica. Porto Alegre: Artmed, 2007, p. 3144.

FERREIRA, Norma Sandra de Almeida. As pesquisas denominadas Estado da Arte.

Educação \& Sociedade, v. 23, n. 79, p. 257-272, agosto, 2002.

FRANÇA, Cristineide Leandro; MATA, Karen Weizenmann da; ALVES, Elioenai Dornelles. Psicologia e Educação à distância: uma revisão bibliográfica: Ciência \& Profissão, v. 32, n. 1, p. 4-15, 2012.

GATTI, Bernadete Angelina. A construção da pesquisa em educação no Brasil. Brasília, DF: Liber Livro, 2012.

GONÇALVES, Nadia Gaiofatto. A relação Estado e Educação na produção acadêmica brasileira. Tese (Doutorado em Educação). Faculdade de Educação da USP. São Paulo, 2003.

GOUGH, David. Síntese sistemática de pesquisa. In: THOMAS, Gary; PRING, Richard (Orgs.). Educação baseada em evidências: a atualização dos achados científicos para a qualificação da prática pedagógica. Porto Alegre: Artmed, 2007, p. 57-78.

GREGÓRIO, Marcia Gomes. Os estudos de educação comparada internacional no banco de dissertações e teses da Capes no período de 1987 a 2006. Dissertação (Mestrado em Educação). Universidade Federal de São Carlos. São Carlos, 2009.

GRELLO, Fabiola Bouth. NUPES: Protagonista de uma história recente da política para educação superior brasileira? (1989-2005). Dissertação (Mestrado em Educação). Universidade Federal de São Carlos. São Carlos, 2008.

JACOMINI, Márcia Aparecida. Avaliação da aprendizagem em tempos de progressão continuada: o que mudou? Um estudo de teses e dissertações sobre o tema (2000-2010). Ensaio: Avaliação e Políticas Públicas em Educação, Rio de Janeiro, v. 22, n. 84, p. 807-828, jul./set. 2014.

MAIA, Graziela Zambão Abdian. As publicações da Anpae e a trajetória do conhecimento em educação no Brasil. Tese (Doutorado em Educação). Faculdade de Filosofia e Ciências, Universidade Estadual. Marília, 2004. 
MAINARDES, Jefferson; TELLO, César. A pesquisa no Campo da Política Educacional: explorando diferentes níveis de abordagem e abstração. Arquivos Analíticos de Políticas Educativas, 24 (75), p. 1-17, 2016. Disponível em:

<http://dx.doi.org/10.14507/epaa.24.2331>. Acesso em: 25/02/2017.

MARCELO, Julia Fernandes; HAYASHI, Maria Cristina Piumbato Innocentini. Estudo bibliométrico sobre a produção científica no campo da sociologia da ciência. Inf. Inf., Londrina, v. 18, n.3, p. 138-153, set./dez. 2013.

MARTINS, Ângela Maria (Org.). Estado da arte: gestão, autonomia escolar e órgãos colegiados (2000-2008). Brasília: Liber Livro, 2011.

MAUÁ JUNIOR, Reynaldo. Planejamento escolar: um estudo a partir de produções acadêmicas (1961-2005). Tese (Doutorado em Educação). Universidade Estadual Paulista. Marília, 2007.

MIRANDA, Leticia Miranda de; FARIAS, Sidney Ferreira. As contribuições da internet para o idoso: uma revisão da literatura: Interface: Comunicação, Saúde, Educação, v. 13, n. 29, p. 383-395, abr./jun. 2009.

MORAES, M. C. M. Recuo da Teoria: dilemas na pesquisa em educação. Revista Portuguesa de Educação, Braga, vol. 14, n. 1, p. 7-25, 2001.

MORAIS, Jaqueline de Fátima dos Santos; ASSUMPÇÃO, Renato Poubel de Sousa. Olhares para a produção bibliográfica sobre educação física escolar: algumas reflexões a partir de um levantamento bibliográfico. Acta Scientiarum Education, Maringá, v. 34, n. 1, p. 121-128, 2012.

NOBREGA-THERRIEN, Sílvia Maria; THERRIEN, Jacques. Trabalhos científicos e o estado da questão: reflexões teórico-metodológicas. Estudos em Avaliação Educacional, v. 15, n. 30, p. 5-16, jul./dez. 2004.

OLIVEIRA, Maria Teresa Cavalcanti de. A Educação Ideológica no Projeto de Desenvolvimento Nacional do ISEB (1955-1964). Tese (Doutorado em Educação). Pontifícia Universidade Católica do Rio de Janeiro. Rio de Janeiro, 2006.

PROST, Antoine. As palavras. In: RÉMOND, René. (Org.) Por uma história política. Rio de Janeiro: Editora UFRJ, 1996. p. 295-330.

SANTOS, Lucas Moreira dos Anjos. Panorama das pesquisas sobre TDIC e a formação de professores de língua inglesa em LA: um levantamento bibliográfico a partir da base de dissertações/teses da Capes. Revista Brasileira de Linguística Aplicada, Belo Horizonte, v. 13, n. 1, p. 15-36, 2013.

SOBRAL, Fernanda Ribeiro; CAMPOS, Claudinei José Gomes. The use of methodology in nursing care and teachinh in national productions: na integrative review. Revista da Escola de Enfermagem da USP, São Paulo, v. 46, n. 1, p. 208-2018, 2012.

SOUZA, Celina. Estado do Campo da pesquisa em políticas públicas no Brasil: RBCS, v. 18, n. 51 , p. 15-20, fev. 2003. 
STREMEL, Silvana. A constituição do campo acadêmico da política educacional no Brasil. Tese (Doutorado em Educação). Universidade Estadual de Ponta Grossa. Ponta Grossa, 2016.

TELLO, C.; MAINARDES, J. Revistando o enfoque das epistemologias da política educacional. Práxis Educativa, Ponta Grossa, v. 10, n. 1, p. 153-178, jan./jun. 2015.

THOMAS, Gary. Introdução: evidências e práticas. In: THOMAS, Gary; PRING, Richard (Orgs.). Educação baseada em evidências: a atualização dos achados científicos para a qualificação da prática pedagógica. Porto Alegre: Artmed, 2007, p. 9-30.

VOSGERAU, Dilmeire Sant'Anna Ramos; ROMANOWSKI, Joana Paulin. Estudos de revisão: implicações conceituais e metodológicas. Diálogo Educacional, Curitiba, v. 14, n. 41, p. 165-189, jan./abr. 2014.

WARDE, Mirian. 0 papel da pesquisa na pós-graduação em Educação. Cadernos de Pesquisa, São Paulo, v. 73, p. 67-75, maio 1990.

WITTMANN, Lauro Carlos; GRACINDO, Regina Vinhares (coords.). 0 estado da arte em política e gestão da educação no Brasil 1991 a 1997. Brasília: ANPAE, Campinas:

Editora Autores Associados, 2001.

YANAGUITA, Adriana Inácio. A produção e circulação de saberes sobre financiamento da educação no Brasil (1991-2005). Dissertação (Mestrado em Educação). Faculdade de Filosofia e Ciências, Universidade Estadual Paulista. Marília-SP, 2008. 


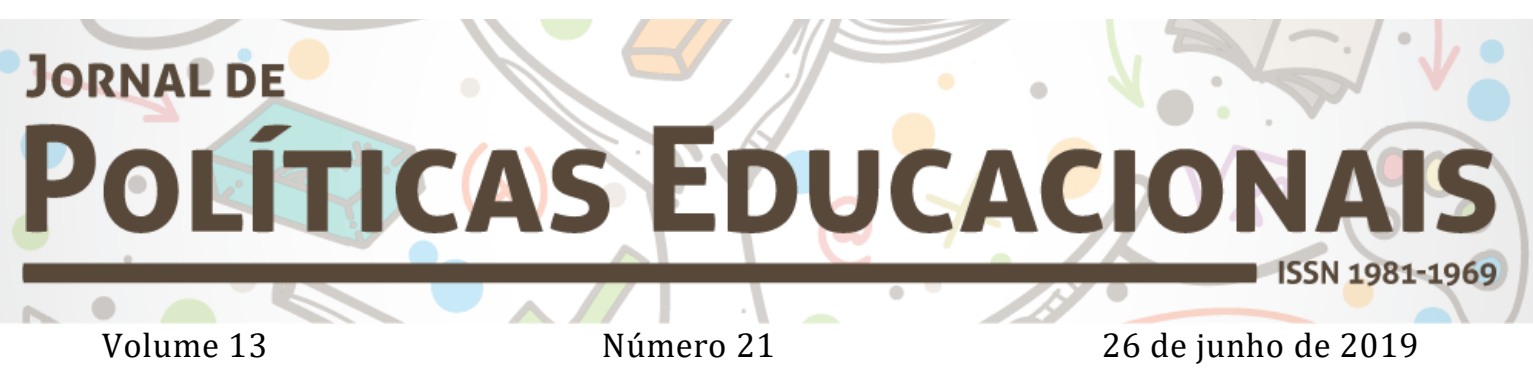

\begin{abstract}
(C)
SOREERIGHIS RESERVED O Copyright é retido pelo/a autor/a (ou primeiro coautor) que outorga o direito da primeira publicação ao Jornal de Políticas Educacionais. Mais informação da licença de CreativeCommons encontram-se em http://creativecommons.org/licenses/by-nc-nd/2.5. Qualquer outro uso deve ser aprovado em conjunto pelo/s autor/es e pelo periódico.

JoRnAl DE Políticas EduCACIONAIS é uma publicação do Núcleo de Políticas Educacionais do Setor de Educação da Universidade Federal do Paraná - NuPE/UFPR, em consórcio com a Linha de Pesquisa em Políticas Educacionais do Programa de Pós-Graduação em Educação - PPGE/UFPR, que aceita colaboração, reservando-se o direito de publicar ou não o material espontaneamente enviado à redação. As colaborações devem ser enviadas ao NuPE/UFPR, conforme orientações contidas nas páginas do periódico na internet: http://revistas.ufpr.br/jpe.
\end{abstract}

\author{
Indexação: \\ BBE - Biblioteca Brasileira de Educação (MEC/INEP) \\ Clase (Base de Datos Bibliográfica de Revistas de Ciencias Sociales y Humanidades) \\ Diadorim - Diretório de Política de Acesso Aberto das Revistas Científicas Brasileiras (IBICT) \\ Google Scholar \\ Index Copernicus \\ Portal de Periódicos (CAPES) \\ SER - Sistema Eletrônico de Revistas da Universidade Federal do Paraná (SER/UFPR) \\ Sumários de Revistas Brasileiras (FUNPEC-RP) \\ DRJI - Directory of Research Journals Indexing
}

(Periódico integralmente disponível apenas em via eletrônica)

Jornal de Políticas Educacionais / Núcleo de Políticas Educacionais da Universidade Federal do Paraná NuPE/UFPR - v.1, n. 1 (1ํs semestre de 2007) - Curitiba: NuPE/UFPR.

Volume 13, número 21 - Junho de 2019

ISSN 1981-1969

1. Educação - Periódicos. 2. Política Educacional - Periódicos. I. NuPE/UFPR

Comitê Editorial:

Elisângela Scaff (UFPR)

Daniela de Oliveira Pires (UFPR)

Ana Lorena Bruel (UFPR)

Conselho Editorial:

Andréa Barbosa Gouveia (UFPR), Ângela Hidalgo (UNICENTRO), Cesar GernominoTello (Universidad Nacional TresFebrero, Argentina),Gladys Beatriz Barreyro (USP), Juca Gil (UFRGS), Jefferson Mainardes (UEPG), João Ferreira de Oliveira (UFG), Luiz Souza Júnior (UFPB), Marcos Edgard Bassi (UFSC), Regina 
JACOMINI, M. A.; PENNA, M. G. de O.; BELLO, I. M. Estudos de revisão sobre produção acadêmica em políticas educacionais (2000-2010)

Maria Michelotto (UFPR), Robert Verhine (UFBA), Rosana Cruz (UFPI), Rubens Barbosa Camargo (USP), Sebastián Donoso Díaz (Universidad de Talca, Chile), Taís Moura Tavares (UFPR), TheresaAdrião (UNICAMP), Vera Peroni (UFRGS).

Créditos e Agradecimentos:

Revisão de Língua Portuguesa, Abstract e Resumen: PROGRAMA DE APOIO ÀS PUBLICAÇÕES CIENTÍFICAS PERIÓDICAS DA UFPR

Arte e diagramação: TIAGO TAVARES (tiagotav@gmail.com)

\author{
Jornal de Políticas Educacionais \\ Universidade Federal do Paraná \\ Setor de Educação \\ Núcleo de Políticas Educacionais - NuPE/UFPR \\ Avenida Sete de Setembro, 2645 \\ 2 andar, Sala 213 \\ 80.230-010 - Curitiba - PR - Brasil \\ Tel.: 41-3535-6264 \\ jpe@ufpr.br \\ http://revistas.ufpr.br/jpe
}

